\title{
Glossopharyngeal Neuralgia Due to Vertebrobasilar Dolichoectasia Coexisting with Fusiform Aneurysm of the Basilar Artery
}

\author{
Jianguo Zhong1, Zhengyu Dai2*, Fei Chen2, Gendi Wang1, Haicun Shi', Weigeng Song1, \\ Congsong Dong2 \\ ${ }^{1}$ Department of Neurology, The Affiliated Yancheng Hospital of Southeast University Medical College, \\ Yancheng, China \\ ${ }^{2}$ Department of Radiology, The Affiliated Yancheng Hospital of Southeast University Medical College, \\ Yancheng, China \\ Email: ${ }^{*}$ shuibin1988@163.com, ${ }^{*}$ ycsydzy@163.com
}

Received 11 August 2014; revised 10 September 2014; accepted 5 October 2014

Copyright (C) 2014 by authors and Scientific Research Publishing Inc.

This work is licensed under the Creative Commons Attribution International License (CC BY). http://creativecommons.org/licenses/by/4.0/

(c) (i) Open Access

\begin{abstract}
Glossopharyngeal neuralgia (GPN) refers to paroxysmal severe pain located to glossopharyngeal part or ear, which is a rare clinical event. In this report, we describe a patient suffered from glossopharyngeal neuralgia due to vertebrobasilar dolichoectasia (VBD). A 72-year-old Chinese male was referred to our clinic with a complaint of paroxysmal severe pain in the right external auditory canal and auricle since 1 month, which could be precipitated by feed and accompanied with the vagus nerve irritation (bradycardia, low blood pressure, syncope) sometimes. Both computed tomographic angiography and digital subtraction angiography demonstrated a VBD (maximum diameter $5.7 \mathrm{~mm}$ ) and a large fusiform aneurysm of basilar artery (maximum diameter $\mathbf{1 3 . 8} \mathbf{~ m m}$ ). The diagnosis is established on clinical and radiological signs. This is the first report of this kind in the literature. Although a few cases demonstrate that VBD can cause GPN, it should be considered as a possible etiology in patients with GPN.
\end{abstract}

\section{Keywords}

Glossopharyngeal Neuralgia, Vertebrobasilar Dolichoectasia, Computed Tomographic Angiography, Digital Subtraction Angiography

\footnotetext{
*Corresponding authors. The two authors contributed equally to this work.
} 


\section{Introduction}

Glossopharyngeal neuralgia (GPN) refers to paroxysmal severe pain located to glossopharyngeal part and ear, which is a rare clinical event [1]. Vertebrobasilar dolichoectasia (VBD) is characterized by marked elongation, dilatation and tortuosity of the vertebral and the basilar arteries. Although VBD is frequently asymptomatic, it may be accompanied with clinical manifestations, such as posterior circulation stroke, intracranial bleeding, cranial nerve disorders, compression of brainstem or obstructive hydrocephalus [2]. To our knowledge, this is the first reported case of GPN due to VBD with no other cranial nerve involvement. In this report, we describe a patient suffered from GPN due to VBD coexisting with fusiform aneurysm of basilar artery.

\section{Case Report}

A 72-year-old Chinese male, presented with a 10-year history of hypertension, was referred to our clinic with a complaint of paroxysmal severe pain in the right external auditory canal and auricle since 1 month. His hypertension was not well controlled for irregular medical treatment. Neurological examination revealed lancinating pain lasting for a few seconds that could be provoked by food arriving at right pharyngeal region after chew. The pain usually attacked in a frequency ranged from 10 to 20 times every day. The neurological examination was otherwise unremarkable.

Four days after the onset, paroxysmal pain attack, last for approximate one hour, accompanied with chest distress, sweating, nigrescent vision and short confusion of consciousness (about $1 \mathrm{~min}$ ). During the attack, the lowest heart rate was 20 times per minute and the blood pressure decreased to 50/30 mmHg approximately. At the same day, he had consulted doctors at a local hospital and had been diagnosed to sick sinus syndrome. Two days later, the old male was operated by permanent pacemaker implantation for his low heart rate. The frequency, intensity and duration time of pain attack were ditto as pre-operation. Ten days prior to hospitalization, the pain frequency gradually increased to more than 20 times every day. Pain frequency and intensity had increased to the extent of interfering with his eating habits and sleep pattern. He could only eat a little liquid food and felt himself weakness. No history of diabetes mellitus was reported and physical examinations were normal.

Magnetic resonance imaging (MRI) can not be examined due to the pacemaker. Both computed tomographic angiography (CTA) and digital subtraction angiography (DSA) demonstrated a marked prolonged and dilated left vertebral artery (maximum diameter $5.7 \mathrm{~mm}$ ) and a large fusiform aneurysm of basilar artery (maximum diameter $13.8 \mathrm{~mm}$ ) (Figure 1). The left vertebral artery wound around the medulla oblongata to the right and impinged on the right medulla oblongata in the root exit zone for the glossopharyngeal and vagus nerve (Figure 1(A) and Figure 1(B)). The enlarged basilar artery then wound around the brainstem to reach the midline before it bifurcated (Figures $1(\mathrm{C})-(\mathrm{F})$ ).

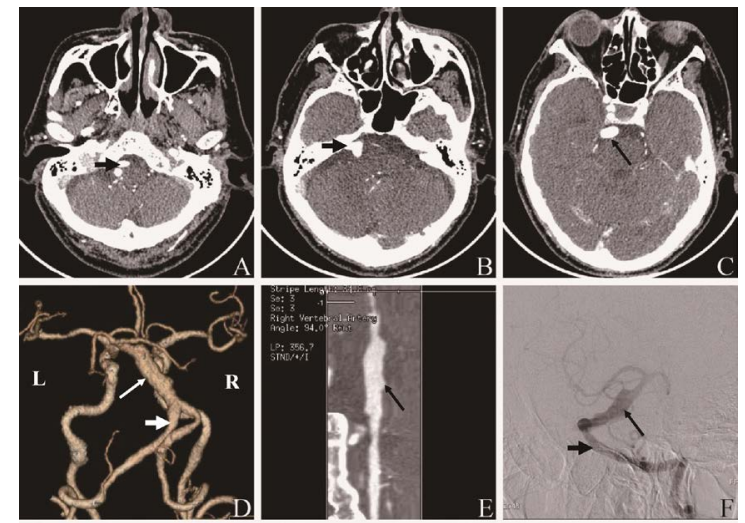

Figure 1. The manifestation of computed tomographic images, computed tomographic angiography and digital subtraction angiography. Gadolinium-enhanced computed tomographic images ((A), (B) and (C)) showing vertebrobasilar dolichoectasia (VBD) impinged on the right medulla oblongata in the root exit zone for the glossopharyngeal and vagus nerve (short arrow) (A) and fusiform aneurysm (long arrow) (C). Computed tomographic angiography of volume rendering image (D) and curved planar reformations image (E) and digital subtraction angiography (F) showing VBD (short arrow) and the fusiform aneurysm (long arrow). 
Surgery was not considered as the first line of treatment due to his unstable hypertension and the permanent pacemaker implantation. In our hospital, he was started on low dose carbamazepine, the dose was gradually escalated to $400 \mathrm{mg}$ per day (200 mg bid). One week after hospitalization, the paroxysmal pain was alleviated obviously. The frequency of pain attack was 1 to 2 times per day, and the duration was 1 to 2 seconds. Then, patient asked for out-patient therapy. At his first follow up visit (one month after hospitalization), he reported the frequency and the intensity of pain was stable and his hypertension was well controlled. Further follow up is continuing.

\section{Discussion}

GPN is a rare disease with wide range of aetiologies. The morbidity of GPN is $0.2-0.4 / 100,000$ person-years [1]. The causes of GPN include tumor, infection, Chiari malformation, infarction and vascular compression, and so on [3]-[6]. The clinical manifestation of GPN is unilateral glossopharyngeal or auditory paroxysmal severe lancinating pain, which can be provoked by swallowing, chewing and cough. Sometimes, the pain could be bilateral or accompanied with bradycardia, syncope and cardiac arrest [5].

VBD is a radiological diagnosis. The mean diameter of the normal basilar artery is $3.17 \mathrm{~mm}$ at the level of the pons [7]. Widely accepted criteria for diagnosis of VBD are an arterial diameter of over $4.5 \mathrm{~mm}$ at any location along its course and deviation of any portion by over $10 \mathrm{~mm}$ from the shortest expected course [8]. Estimates of prevalence of VBD range between $0.2 \%$ and $4.4 \%$, but sound population-based data are lacking [2]. The longterm complications of VBD mainly include ischemic stroke, compression of brainstem and cranial nerves, transient ischemic attack, hemorrhagic stroke, hydrocephalus, and subarachnoid hemorrhage [2]. The most common involved cranial nerves are facial and trigeminal nerve, which result in facial spasm or trigeminal neuralgia or both them [1]. To our knowledge, this is the first reported case of VBD compression of the root exit zone for the glossopharyngeal and vagus nerve with no other cranial nerve involvement.

Treatment of VBD is in a dilemma. Treatment options include no treatment, treatment with platelet aggregation inhibitors or anticoagulants, (micro) surgery or endovascular treatment [2]. The management of such a patient is not well defined. Medical management would be the first line of treatment. Symptoms caused by compression of cranial nerve may be alleviated by decompressive surgery, a procedure that is associated with a significant risk of embolic stroke in patients with VBD. Our patient remains free of any other neurological symptoms to this date. As surgical management is not recommended, his condition remains untreatable. He continues to be followed up on medical management.

\section{Conclusion}

In conclusion, we described a patient afflicted with GPN, which could be precipitated by feed and accompanied with the vagus nerve irritation (bradycardia, low blood pressure, syncope) sometimes, together with a VBD detected by CTA and DSA. Although a few cases demonstrate that VBD can cause GPN, it should be considered as a possible etiology in patients with GPN.

\section{Acknowledgements}

We are grateful to our patient and his parents, who agreed to the publication of this case report and provided all the information we needed.

\section{References}

[1] Van Hecke, O., Austin, S.K., Khan, R.A., Smith, B.H. and Torrance, N. (2014) Neuropathic Pain in the General Population: A Systematic Review of Epidemiological Studies. Pain, 155, 654-662. http://dx.doi.org/10.1016/j.pain.2013.11.013

[2] Wolters, F.J., Rinkel, G.J. and Vergouwen, M.D. (2013) Clinical Course and Treatment of Vertebrobasilar Dolichoectasia: A Systematic Review of the Literature. Neurological Research, 35, 131-137. http://dx.doi.org/10.1179/1743132812Y.0000000149

[3] Phuong, H.L., Matsushima, T., Hisada, K. and Matsumoto, K. (2004) Glossopharyngeal Neuralgia Due to an Epidermoid Tumour in the Cerebellopontine Angle. Journal of Clinical Neuroscience, 11, 758-760. http://dx.doi.org/10.1016/j.jocn.2003.07.013 
[4] Warren, H.G., Kotsenas, A.L. and Czervionke, L.F. (2006) Trigeminal and Concurrent Glossopharyngeal Neuralgia Secondary to Lateral Medullary Infarction. American Journal of Neuroradiology, 27, 705-707.

[5] Rey-Dios, R. and Cohen-Gadol, A.A. (2013) Current Neurosurgical Management of Glossopharyngeal Neuralgia and Technical Nuances for Microvascular Decompression Surgery. Neurosurgical Focus, 34, E8.

[6] Ruiz-Juretschke, F., Garcia-Leal, R., Garcia-Duque, S., Panadero, T. and Aracil, C. (2012) Glossopharyngeal Neuralgia in the Context of a Chiari Type I Malformation. Journal of Clinical Neuroscience, 19, 614-616. http://dx.doi.org/10.1016/j.jocn.2011.05.040

[7] Smoker, W.R., Price, M.J., Keyes, W.D., Corbett, J.J. and Gentry, L.R. (1986) High-Resolution Computed Tomography of the Basilar Artery: 1. Normal Size and Position. American Journal of Neuroradiology, 7, 55-60.

[8] Smoker, W.R., Corbett, J.J., Gentry, L.R., Keyes, W.D., Price, M.J. and McKusker, S. (1986) High-Resolution Computed Tomography of the Basilar Artery: 2. Vertebrobasilar Dolichoectasia: Clinical-Pathologic Correlation and Review. American Journal of Neuroradiology, 7, 61-72. 
Scientific Research Publishing (SCIRP) is one of the largest Open Access journal publishers. It is currently publishing more than 200 open access, online, peer-reviewed journals covering a wide range of academic disciplines. SCIRP serves the worldwide academic communities and contributes to the progress and application of science with its publication.

Other selected journals from SCIRP are listed as below. Submit your manuscript to us via either submit@scirp.org or Online Submission Portal.
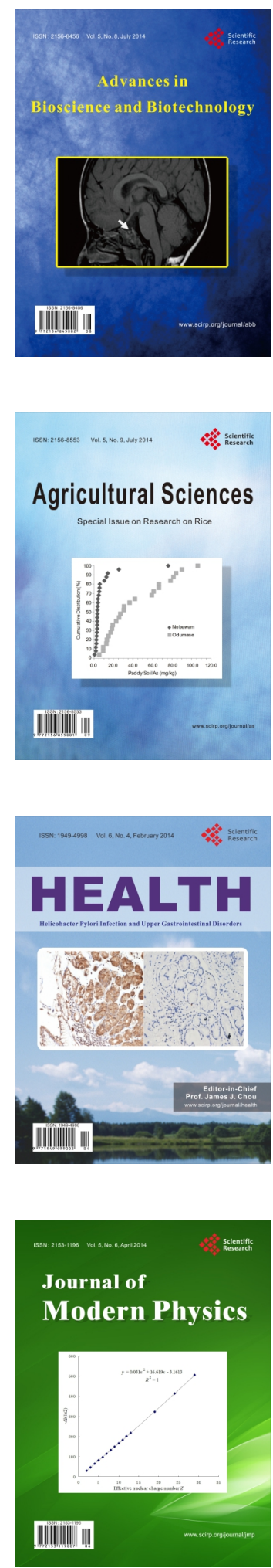
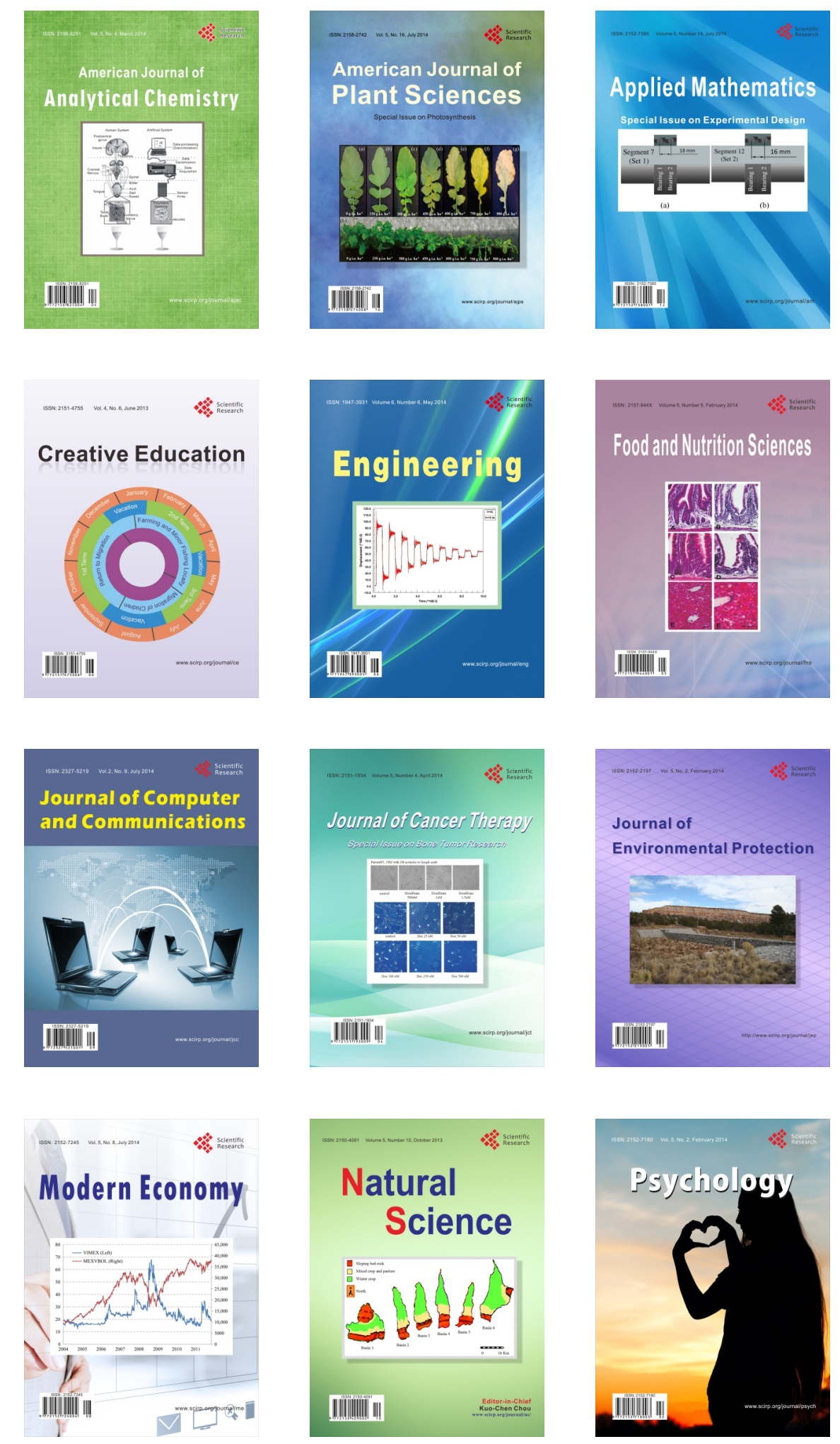